\title{
Development and Optimization of a High Titer Recombinant Lentivirus System
}

Austin Storck, James Ludtke, Leisha Kopp, and Laura Juckem*

Mirus Bio LLC, Madison, Wisconsin USA

\section{Abstract}

To enable simple and effective high titer recombinant lentivirus production, we examined key parameters for the generation of lentivirus including: transfection optimization, media change, incubation time and DNA vector selection. These results illustrate the importance of optimizing transfection processes for high titer recombinant lentivirus production.

\section{Introduction}

Lentivirus is an enveloped single-stranded RNA virus from the Retroviridae family. Its ability to infect dividing and non-dividing cells has led to the widespread utilization of recombinant lentivirus as a gene delivery vehicle. In addition, lentivirus has an efficient integration mechanism which leads to robust and stable transgene expression in target cells.

Recombinant lentivirus production is accomplished in human embryonic kidney derived cells that stably express the SV40 large T antigen (e.g. 293T/17 cells). These cells are transiently transfected with three or four plasmids that encode the gene of interest (GOI) and the essential viral genes: gag, pol, and rev, along with the vesicular stomatitis virus envelope protein $G$ (VSV-G). Once all the genes are expressed at sufficient levels the virus is assembled and buds through the plasma membrane to form an enveloped virion pseudotyped with VSV-G which conveys the ability to transduce a broad range of mammalian cell types. The viruscontaining supernatant can be collected and filtered to remove any cells.

\section{Cell Confluency}

A key parameter for successful transfection and subsequent virus production is the cell confluency at the time of transfection. Low confluency can lead to cytotoxicity and lower transfection efficiency. Performing transfections at high confluence, e.g. 85-90\%, will maximize the protein expression levels and subsequent titers.

\section{Reagent-to-DNA Ratio}

The transfection reagent-to-DNA ratio is a critical parameter of efficient nucleic acid delivery. The positively charged transfection reagent must be supplied in sufficient quantities to effec-

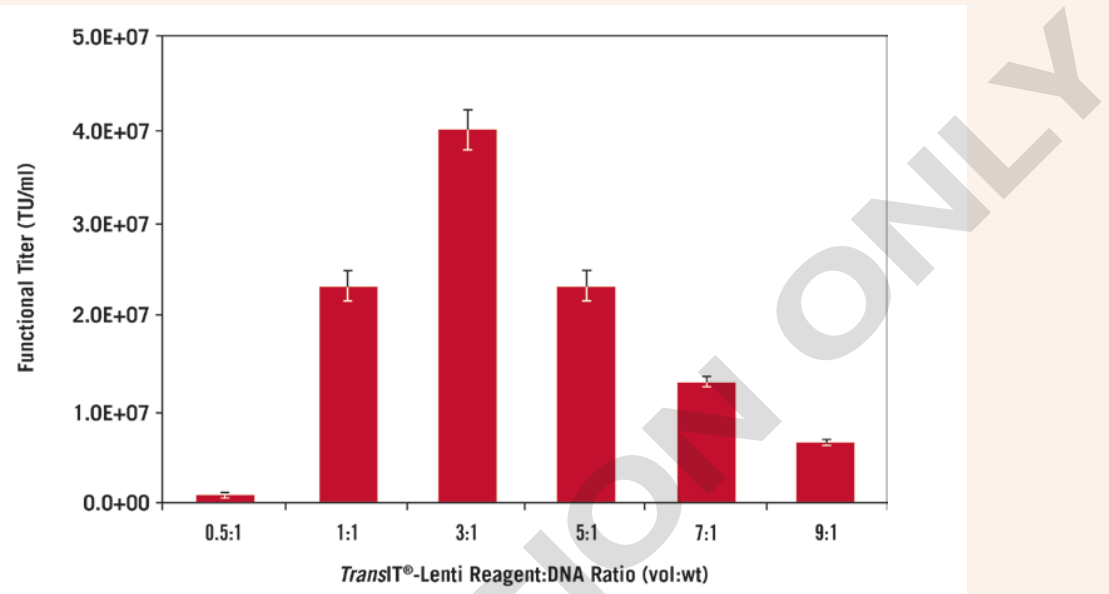

Figure 1. Optimization of Reagent-to-DNA Ratios. Adherent 293T/17 cells were transfected in a 12-well plate format using the vectors (pLKO.1-puro-CMV-TurboGFPTM transfer vector and the Lentivirus Packaging Mix, Powered by MISSION ${ }^{\circledR}$ (Mirus Bio) using the TransI ${ }^{\circledR}$-Lenti Transfection Reagent (Mirus Bio) at varying reagent-to-DNA ratios (0.5:1-9:1). The supernatant was harvested, filtered $(0.45 \mu \mathrm{m})$, and titered using 293T/17 cells. Lentivirus transductions were performed in the presence of $8 \mu \mathrm{g} / \mathrm{ml}$ TransducelT TM (Mirus Bio) and GFP expression was measured 72 hours post-transduction using Guava ${ }^{\circledR}$ easyCyte ${ }^{\mathrm{TM}}$ 5HT Flow Cytometer (Millipore Sigma). Error bars represent the range of duplicate wells. Functional titers were calculated using virus dilutions with less than $20 \%$ GFP positive cells.

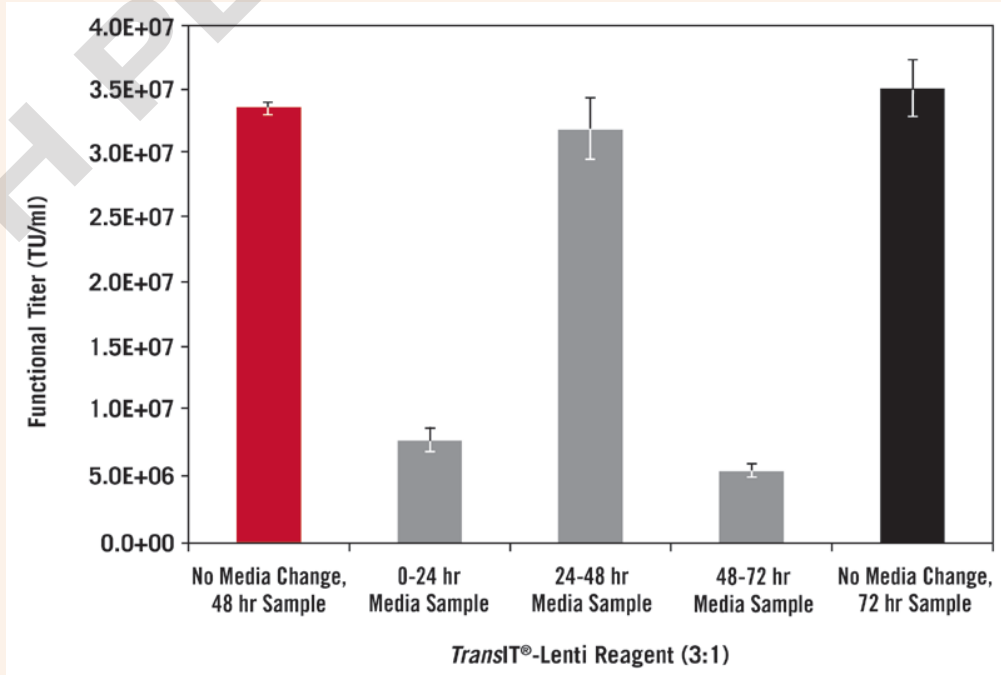

Figure 2. Single Harvest and No Media Change Required. Adherent 293T/17 cells were transfected in a 6-well plate with pLKO.1-puro-CMV-TurboGFPTM transfer vector and the Lentivirus Packaging Mix Powered by MISSION ${ }^{\circledR}$ (1:1 ratio, $2 \mu \mathrm{g} /$ well, Mirus Bio) with the TransI ${ }^{\circledR}{ }^{-}$Lenti Transfection Reagent (Mirus Bio) at a 3:1 reagent-to-DNA ratio (vol:wt), The supernatant was harvested at the indicated time points, filtered $(0.45 \mu \mathrm{m})$, and titered using 293T/17 cells. Lentivirus transductions were performed in the presence of $8 \mu \mathrm{g} / \mathrm{ml}$ TransducelT ${ }^{\mathrm{TM}}$ (Mirus Bio) and GFP expression was measured 72 hours post-transduction using Guava ${ }^{\circledR}$ easyCyte ${ }^{\mathrm{TM}} 5 \mathrm{HT}$ Flow Cytometer (Millipore Sigma). Error bars represent the standard range of duplicate wells titered individually. Functional titers were calculated using virus dilutions with less than $20 \%$ GFP positive cells. 
tively condense and coat the negatively charged plasmid DNA. For ease of use, we formulated the TransIT ${ }^{\circledR}$-Lenti Transfection Reagent to work at a 3:1 reagentto-DNA ratio (volume: weight). Altering the reagent-to-DNA ratio can adversely affect the virus titers (Figure 1). Similarly, we found a total plasmid concentration of $1 \mu \mathrm{g} / \mathrm{ml}$ of culture works best with the TransI ${ }^{\circledR}{ }^{\circledR}$-Lenti Transfection Reagent.

The order of addition, composition and stoichiometry of the plasmids can greatly influence the titers obtained. Depending on the virus generation, three or four plasmids need to be premixed prior to adding to the complex to ensure that the transfection complexes are not formed preferentially with one plasmid over another. In addition, if using individual packaging plasmids we recommend a starting ratio of 6:4:1:1 of transfer:gagpol:rev:VSV-G vectors. If using a lentivirus packaging premixture we recommend an initial ratio of 1:1 between the packaging mixture and the transfer plasmid.

\section{Media Change Post-Transfection}

The harvest procedure during recombinant lentivirus production often includes a media change 24 hours posttransfection, as well as, multiple harvests at 48 and 72 hours post-transfection. To investigate the importance of these parameters we tested the functional lentivirus titers in media samples post-transfection, as well as, undisturbed at 48 and 72 hours post-transfection. By measuring the functional titers obtained from the media samples we found that the majority of the recombinant lentivirus was produced between 24-48 hours post-transfection (Figure 2). We also observed no difference in the functional lentivirus titers when the virus is harvested at 48 or 72 hours post-transfection without a media change suggesting that this step can be omitted without adverse affects. These findings demonstrate a workflow that reduces experimental complexity and workload.

\section{Transfection Reagent Selection}

High efficiency transfection is necessary, but not sufficient for high titer recombinant lentivirus production. To empirically determine the best transfection formulation for lentivirus production we initially screened our compound libraries using a reporter assay system; however, when we compared the reporter assay output with functional lentivirus production we did not always observe a correlation. Therefore, we employed a targeted compound screening approach to directly test the effect of reagent formulation on functional lentivirus titers. During this process we identified a novel formulation, the Transl ${ }^{\circledR}{ }_{-}$Lenti Transfection Reagent, which yields up to 8-fold higher functional titers in head-to-head comparisons with other commonly used transfection technologies (Figure 3).

\section{Plasmid DNA}

Vector design is a critical factor in recombinant lentivirus production. Advances in the safety of lentivirus production has been accomplished through the deletion of non-essential components of the virus genome, in addition to, separation of essential elements onto different plasmid vectors. In addition, the composition and design of the plasmid vector greatly affects the overall virus titer.

Transfer vectors encoding the GOI can be swapped while maintaining the same packaging vectors. In studying three different commercially available third generation transfer vectors we observed up to a 4-fold difference in lentivirus titers (Figure 4a). When performing system-to-system comparisons with different transfer vectors, packaging mixes and transfection reagents the results are compounded and result in a combined 100-fold difference between commercially available systems (Figure 4b). This illustrates the synergy between the essential components of recombinant lentivirus production. 
A.

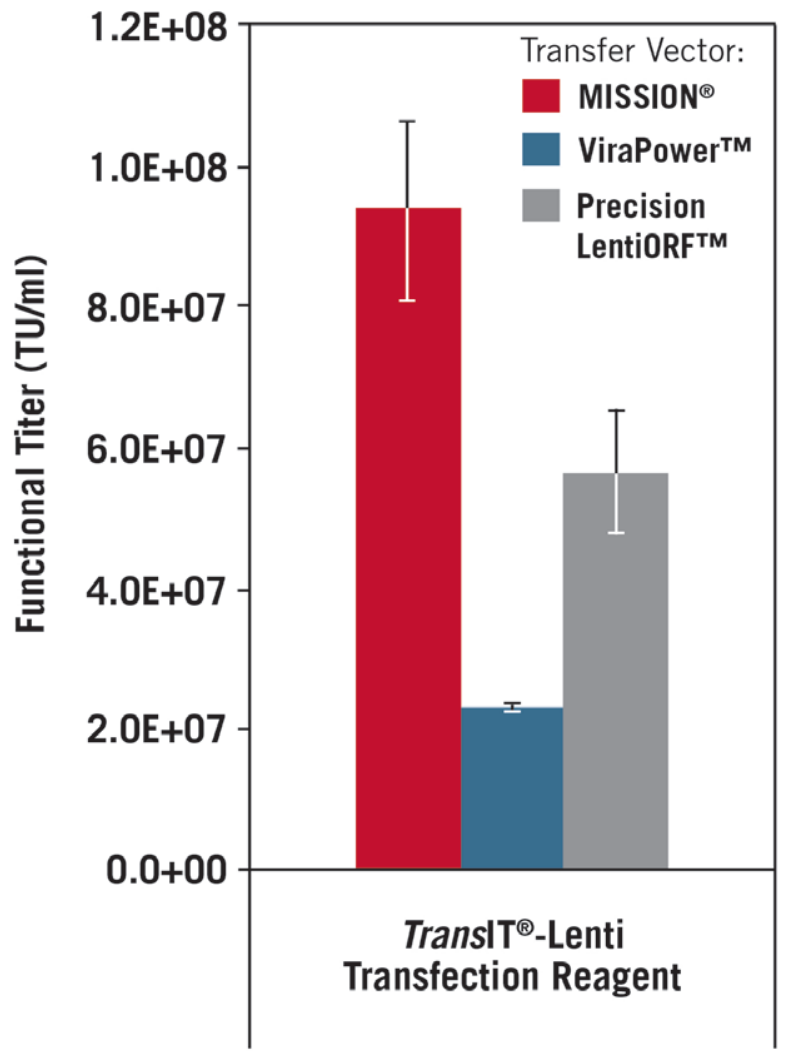

B.

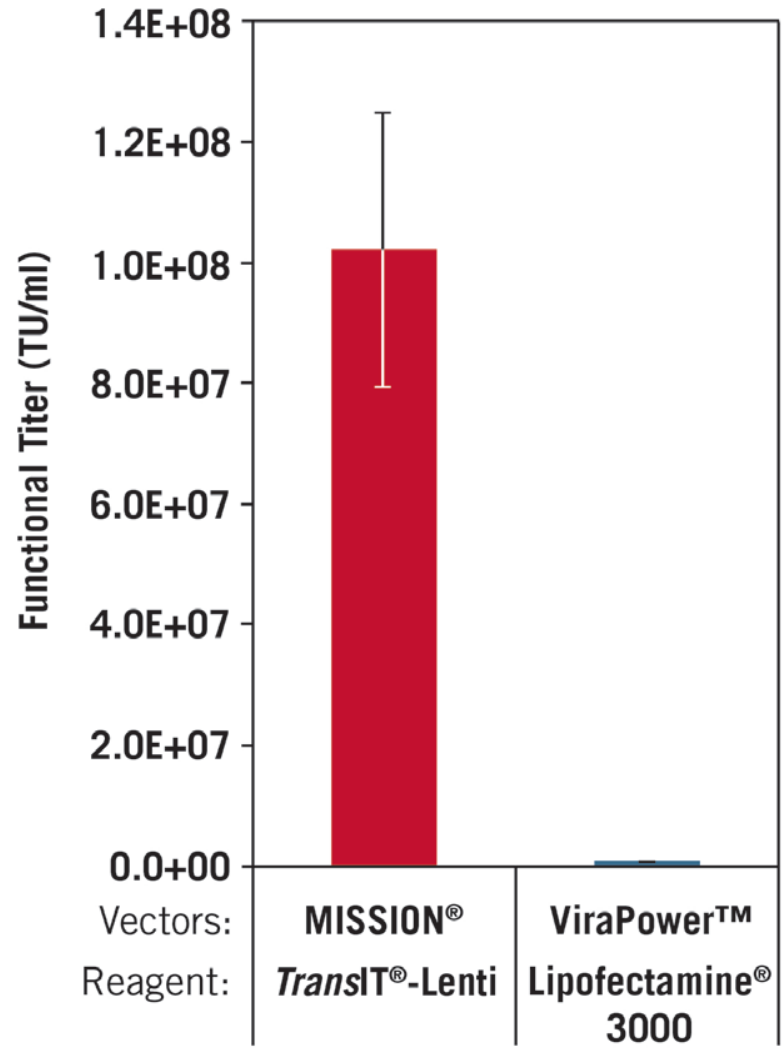

Figure 4. Vector Selection Greatly Affects Lentivirus Titer. (A) Adherent 293T/17 cells were transfected in a 6-well plate with one of three transfer vectors: MISSION ${ }^{\circledR}$ pLKO.1-puro-CMV-TurboGFPTM (Millipore Sigma), ViraPower ${ }^{\mathrm{TM}}$ pLenti6.2-GW/EmGFP (Thermo Fisher Scientific), or Precision Lenti-ORFTM RFP (GE Life Sciences), and the Lentivirus Packaging Mix Powered by MISSION ${ }^{\circledR}$ Genomics (total $2 \mu \mathrm{g} /$ well, MirusBio) using the TransIT ${ }^{\circledR}$-Lenti Transfection Reagent (3:1, vol:wt, Mirus Bio). or (B) System-to-system were performed using either MISSION ${ }^{\circledR}$ vectors ( $\mathrm{pLKO.1-}$ puro-CMV-TurboGFPTM and Lentiviral Packaging Mix (Millipore Sigma) and the TransIT ${ }^{\circledR}$-Lenti Transfection Reagent or ViraPower ${ }^{\mathrm{TM}}$ vectors (pLenti6.2-GW/EmGFP and Lentiviral Packaging Mix (Thermo Fisher Scientific) and the Lipofectamine ${ }^{\circledR} 3000$ Transfection Reagent (Thermo Fisher Scientific) according to the manufacturers protocol. The supernatant was harvested, filtered $(0.45 \mu \mathrm{m})$, and titered using $293 \mathrm{~T} / 17 \mathrm{cells}$. Lentivirus transductions were performed in the presence of $8 \mu \mathrm{g} / \mathrm{ml}$ TransducelT TM (Mirus Bio) and GFP was measured 72 hours post-transduction using Guava ${ }^{\circledR}$ easyCyte ${ }^{\mathrm{TM}}$ 5HT Flow Cytometer (Millipore Sigma). Error bars represent the standard deviation of triplicate transfection complexes titered individually. Functional titers were calculated using virus dilutions with less than $20 \%$ GFP positive cells.

\section{Conclusions}

We systematically optimized the experimental variables surrounding recombinant lentivirus production including: cell confluency, transfection reagent-to-DNA ratio, media change, incubation time, reagent formulation and DNA vectors. The highest titers are achieved when 293T/17 cells are transfected a 90\% confluency using the TransIT ${ }^{\circledR}$-Lenti Transfection Reagent at a 3:1 reagent-to-DNA ratio (vol:wt). A media change is not required post-transfection and a single harvest at 48 hours posttransfection offers convenience and less handling of the cells. In addition, the transfer and packaging plasmid also play a critical role in the titers achieved. The combination of transfection reagent, transfer vector and packaging premix can lead to a 100 -fold difference in titers in head-to-head comparisons. The TransIT ${ }^{\circledR}$-Lenti Transfection System combining the novel Mirus transfection formulation and the MISSION ${ }^{\circledR}$ Lentivirus Packaging Plasmids provides exceptional lentivirus titers, increased flexibility and reduced workload.

For more information about the TransIT ${ }^{\oplus}$-Lenti Transfection Reagent visit: https://www.mirusbio.com/transit-lenti 